\section{ONOMAREDIN}

Revista semestral de lingüística, filología y traducción
PONTIFICIA UNIVERSIDAD CATÓLICA DE CHILE FACULTAD DE LETRAS

\title{
Sur la notion linguistique de vicariance
}

On the linguistic notion of vicariance

\section{Samuel Bidaud}

Université de Bourgogne

Francia

ONOMÁZEIN 28 (diciembre de 2013): 29-41 DOI: 10.7764/onomazein.28.12

\section{(c) $(i)$}

Samuel Bidaud: Département de Lettres / Philosophie, Université de Bourgogne. Correo electrónico: samuel.bidaud@aliceadsl.fr 


\section{Résumé}

Nous proposons ici une définition de la notion de vicariance. En effet, les grammairiens français qui utilisent ce concept ne l'ont pas défini de façon précise. Nous verrons quelle est la spécificité des mots vicariants, qui peuvent être définis comme des mots avec un sémantisme abstrait qui sont sémantiquement identiques avec leur antécédent.

Mots clés: vicariance; anaphore; verbe faire; conjonction que.

\section{Abstract}

We try here to propose a definition of the notion of vicariance. Indeed, the French grammarians who use this concept have not defined it precisely. We will see the specificity of the vica- riant words, which can be defined as words with an abstract sense which are semantically identical with their antecedent.

Keywords: vicariance; anaphora; verb faire; conjunction que. 


\section{Introduction}

Nous voudrions étudier ici la notion linguistique de vicariance, laquelle n’a pas encore été définie à notre connaissance. Dans cette perspective, nous nous proposons de reconstituer I'historique du terme vicariance et de voir qu'en réalité cette notion se doit d'être élargie à certains termes que la tradition grammaticale ne regroupe pas dans cette classe, à vrai dire très restreinte puisque l'on y trouve généralement seulement le verbe faire et la conjonction que. C'est en nous appuyant sur les propriétés communes de ces deux mots que nous pourrons redéfinir la vicariance dans une mesure moins réductrice.

\section{Historique du terme}

Il n'est pas mauvais de commencer notre délimitation de cette notion de vicariance par un rappel étymologique. Si l'on ouvre les dictionnaires, on peut lire des définitions de la vicariance qui ne sont pas d'ordre grammatical et qui ne reprennent pas nécessairement en outre le mot vicariance lui-même. Ainsi lit-on dans le Grand Larousse de la langue française que l'adjectif vicariant est attesté dès 1878 et qu'il "se dit de l'organe capable de suppléer à l'insuffisance fonctionnelle d'un autre organe" (1978 : 6461).

L'adjectif vicariant est dérivé du mot vicaire, c'est-à-dire "le prêtre qui aide et remplace éventuellement le curé", selon le Nouveau Petit Robert (2008 : 2705); du sens de substitution dans l'office religieux, on est passé à celui de remplaçant en biologie. Resterait à savoir si c'est la biologie qui a donné son nom à la vicariance grammaticale ou si c'est le mot vicaire au sens religieux. On peut sans grand risque, et malgré l'importance de la métaphore biologique dans la linguistique du dix-neuvième siècle, affirmer que c'est du mot vicaire signifiant 'prêtre substitut' qu'est dérivée la notion grammaticale.

Venons-en à présent aux grammairiens.
Comme ces derniers sont peu nombreux à évoquer précisément la vicariance, nous passerons également en revue des termes apparentés au niveau sémantique.

La première attestation du mot vicariance se retrouve probablement chez Maurice Grevisse, dans les Problèmes de langage. Toutefois, Grevisse fait clairement allusion à une tradition grammaticale qui le précède, comme il ressort des lignes qui suivent (1970: 58-59)․․

Si nous nous en rapportons à Littré, faire n'a pas moins de quatre-vingt-deux sens ou emplois particuliers. A Dieu ne plaise que nous nous avisions de les passer en revue! Ce sera bien assez si nous examinons le cas de faire, verbum vicarium (comme parlent les doctes), ou, pour dire en français la chose, substitut d'un verbe qui précède et dont on veut éviter la répétition, comme dans la phrase «je me propose de mieux vivre que je n'ai fait» [= que je n'ai vécu].

Gérard Moignet, dans ses articles sur le verbe faire vicariant en français, utilise une terminologie identique tout en parlant de "suppléance du verbe", et il ajoute (1974: 31):

La fonction de suppléance qu'il assumait (le verbe faire en ancien français) est comparable à celle du mot chose, substantif passe-partout, ou, en langage familier, voire argotique, chose (masc), machin, truc, et, comme on entend aujourd'hui, bidule [...]. On peut aussi rapprocher la fonction de faire, en l'occurrence, de celle de la conjonction que utilisée pour éviter la répétition de quand, si, comme et des conjonctifs dans la composition desquels il entre [...].

Morten Nøjgaard, qui a consacré tout un article au problème du que reprenant si, parle de "coniunctio vicaria” (1970: 125).

Henri Bonnard, dans le Code du français courant (1981: 35), mentionne la vicariance dans la catégorie de la réduction:

Une loi d'économie qui joue dans toutes les langues tend à raccourcir les mots et les chaînes syn-

1 Nous n’avons toutefois trouvé aucune attestation de cette tradition avant Maurice Grevisse. 
taxiques sans diminuer le nombre d'informations communiquées.

[...] Cette réduction se fait :

- soit par l'emploi de représentants, pronoms ou adverbes, qui désignent un référent déjà nommé dans le contexte (anaphore, $\S 12$ ):

Ce livre est pour toi, prends-le (= prends ce livre)

A ce type se rattache l'emploi d'un mot «vicaire» tel que le verbe faire (II a travaillé beaucoup moins que son frère n'avait fait), la conjonction que (Quand il fera jour etque le brouillard sera levé), ainsi que la réponse par oui ou non à une question.

- Soit par la suppression d'un ou plusieurs éléments signifiants dont le signifié est facile à sous-entendre.

L'originalité d'Henri Bonnard est d'ajouter les adverbes oui et non à la liste des mots vicariants, ou du moins de les rapprocher de la vicariance. Désormais, on ne retrouve plus chez les auteurs qui mentionnent la vicariance que le verbe faire, la conjonction que et le mot chose.

Olof Eriksson, qui est le seul auteur à avoir consacré un ouvrage complet à faire vicariant, préfère parler de suppléance verbale, comme Gérard Moignet, que de "pro-verbe" ou de "verbe vicaire", puisque "la représentation ne porte pas normalement [...] sur le seul verbe principal, mais en même temps sur un ou plusieurs autres membres inclus dans la complémentation de ce verbe" (1985: 10).

Georges Kleiber parle de mot "vicaire” (1987: 112) à propos de chose, et évoque également l' "emploi vicarial" de truc, bidule et machin (1987: 113).

Thierry Ponchon définit comme suit le verbe faire vicariant au début du chapitre d'une centaine de pages qu'il consacre à cet emploi en ancien français: "Son pouvoir se borne à être vicariant de tout verbe exprimant une action ou une idée d'action”, et il précise: “(m)ais la limite n’a pas toujours été de cet ordre, notamment en français médiéval" (Ponchon, 1994: 252).

Thomas Verjans, dans l'article qu'il consacre au que vicariant, définit de la manière suivante ce dernier (2007 : 133): "Parmi les multiples emplois du mot que, il en est un, bien connu et communément nommé 'vicariant', qui correspond à la propriété que possède ce terme, en cas de juxtaposition ou de coordination de propositions subordonnées circonstancielles, d'apparaître en tête de la seconde, en lieu et place de la conjonction (ou locution conjonctive) initiale".

Enfin, le Dicionário Houaiss da Língua Portuguesa (2003: 1707) parle, probablement par analogie avec la terminologie française, de verbo vicário et donne comme exemple: gostaria de me expressar como ele o faz ('j'aimerais m'exprimer comme il le fait').

On remarquera d'emblée une chose: peu nombreux sont les auteurs qui emploient le terme de vicariance, et beaucoup préfèrent lui réserver d'autres appellations, qui font néanmoins allusion aux mêmes emplois du verbe faire et de la conjonction que. C'est le cas de Ferdinand Brunot, qui parle dans sa monumentale La pensée et la langue de "verbe substitut" à propos de faire (1936: 17) : "Le verbe substitut faire. - Dès les origines de la langue, on trouve, surtout dans les comparaisons, le verbe convenable remplacé par un verbe de remplacement à tous usages, qui est le verbe de sens général faire[...]”. F. Brunot évoque également le problème du que vicariant, qu'il nomme "représentant de conjonctions", et à propos duquel il écrit (Brunot, 1936 : 706-707):

En m. f., I'usage de remplacer les autres conjonctions par un que se généralisa. Aujourd'hui, au lieu de répéter bien que, quoique, quand on n'a pas intérêt à le faire pour des effets de style, on répète seulement le que contenu dans la locution conjonctive: Je préférerais la tuile, parce qu'elle a l'air plus propre et plus gai que le chaume, qu'on ne couvre pas autrement les maisons dans mon pays, et que cela me rappellerait un peu l'heureux temps dema jeunesse. (ROUSS., Emile, 1, 4). 
[...] D'autre part on remplace des conjonctions diverses par que. Ce remplacement plaisait à Vaugelas. Cette variété n'est point vicieuse, disait-il, mais naturelle de notre langue. Si nous sommes jamais heureux et que la fortune se lasse, est meilleur que : si nous sommes jamais heureux et si... (VAUG, 1, 137; H. L., 3,655).

Louis Hjelmslev, dans l'article qu'il consacre à la nature du pronom, note qu' “il y a [...] des pronoms verbaux exocentriques: c'est dans beaucoup de langues le verbe "faire", qui renferme en un syncrétisme total toutes les significations verbales possibles" (Hjelmslev, 1971 (1937): 206).

B. Pottier développe l'idée d'une catégorie de mots qu'il nomme "super-inclusifs" et à propos desquels il écrit (1967 : 47) : "Les super-inclusifs sont des mots comme truc, machin ou chose, ainsi que les substituts : qui?, quoi?, faire...".

Georges et Robert Le Bidois parlent de "répétition de la ligature" à propos du que vicariant (1968: 440, tome 2):

Où que reprend sa valeur de conjonction, c'est Iorsqu'il représente une ligature exprimée précédemment. Le souci de varier l'expression fait éviter généralement de répéter la locution: "Tant que les hommes pourront mourir et qu'ils aimeront à vivre, le médecin sera raillé et bien payé" La Br. Car XIV, 65 [...]. - Par analogie avec ces constructions, que sert de même à représenter une conjonction temporelle qui ne contient pas que: [...] "Un soir, comme elle venait d'en descendre et qu'il lui disait adieu, elle cueillit..." PROUST Swann II, 9 .

Citons également la Grammaire des langues romanes de Meyer-Lübke, non qu'elle ajoute quoi que ce soit d'un point de vue terminologique ou définitoire, mais parce qu'elle commente le cas de la conjonction vicariante en français et en italien (1900: 730, tome 3):

Lorsqu'on unit entre elles DEUX PROPOSITIONS INTRODUITES PAR LA MÊME CONJONCTION, celleci peut naturellement n'être exprimée qu'une fois; s'agit-il de propositions relatives de généralisation, il est permis en vieux français, au lieu de ki ke... ki ke, ou du simple ki ke, d'employer aussi ki ke... ki [...]. Mais on peut aussi mettre que devant la seconde proposition: cf. en ital. Quando la mattina aprii le persiane e che il più bel sole entrò a illuminare la stanza, i vecchi mobili parvero risvegliarsi (De March., Giac. id. 29).

Il en va de même de la Grammaire des Iangues romanes de Frédéric Diez, citée par Thomas Verjans (2007: 134)

Si la conjonction placée en tête de la proposition accessoire est un mot composé avec que, on ne répète pas la conjonction complète mais seulement le mot de liaison que, auquel est transporté le sens de la conjonction (...). Mais l'espagnol préfère répéter la conjonction entière ou l'omettre tout à fait. Quelques particules simples telles que si, quand, com(m)e peuvent aussi en ce cas être représentées par que; et à ce propos il faut observer que la particule que, lorsqu'elle est prise pour si, se fait suivre en français, et généralement en italien, du subjonctif.

Enfin, signalons que le Grande dizionario della lingua italiana (1964: 29) note que la conjonction che peut être "coordinatta con quando o con una cong. composta con che (con lo stesso significato)" ('coordonnée avec quando ou avec une conjonction composée avec che (avec la même signification)').

Certains mentionnent également la vicariance sans s'y arrêter; tel est le cas d'Olivier Soutet (1992: 71), qui parle d'un "que substitut" en ancien français et qui note la rareté de ce dernier, ou de Sabine Lardon et de Marie-Claire Thomine (2009: 355), qui reprennent la terminologie classique de "faire vicariant" et de "conjonction que vicariante" (2009: 382), pour souligner notamment l'extension du que vicariant en moyen français. D'un point de vue non diachronique, Pierre le Goffic (1993: 532) range le que vicariant dans la catégorie des adverbes connecteurs intégratifs en coordination.

Les définitions que nous avons données suffisent nous semble-t-il en tout cas pour justifier ce qui suit. 
De ces définitions, il ressort tout d'abord le constat que très peu d'auteurs ont traité spécifiquement de la vicariance; en effet, si l'on fait abstraction des articles de Gérard Moignet et de Thomas Verjans, et des livres de Thierry Ponchon et d'Olof Eriksson, la vicariance n'appelle que quelques commentaires rapides, voire quelques lignes. La terminologie prototypique et que nous reprendrons, celle qui théorise sous le concept de vicariance certains emplois de faire et de que en fonction de substitution notamment, regroupe au niveau sémantique la même chose que les termes dérivés comme "verbe substitut" ou autres. A partir de là, la vicariance est parfois élargie à d'autres termes, comme le mot chose chez Gérard Moignet ou oui et non chez Henri Bonnard. II conviendra de voir dans quelle mesure ces ajouts se justifient.

On peut, à partir des définitions qui précèdent, proposer une synthèse. II semble que tous les auteurs soient d'accord pour regrouper dans la vicariance le verbe faire et la conjonction que lorsque ces derniers sont amenés à remplacer un autre verbe ou une autre conjonction, et I'on pourra ajouter que le verbe faire a dans ce cas un sens général, selon la définition de $F$. Brunot. On relèvera que c'est du point de vue de sa fonction de remplacement que la vicariance est définie. Mais alors, plusieurs problèmes se posent à nous. En effet, si c'est la seule fonction de remplacement qui permet de définir la vicariance, qu'est-ce qui différencie cette dernière de la simple anaphore? En est-elle un type particulier? Si oui, quelle est sa singularité, sur laquelle n'insistent aucun des auteurs cités?

Un autre problème concerne les mots vicariants eux-mêmes. On a vu que Gérard Moignet, Georges Kleiber et Henri Bonnard ajoutaient à cette classe des mots comme chose pour les premiers, oui et non pour le second. Dans quelle mesure peut-on rattacher ces mots à la notion de vicariance? Et dans quelle mesure un mot comme oui relèverait-il plus de la vicariance que de l'ellipse?

\section{Problèmes de définition}

Il convient de bien délimiter la vicariance par rapport à l'ensemble des termes avec lesquels elle peut se confondre et entrer en concurrence. C'est à cette fin que nous serons amenés à comparer la vicariance, l'anaphore, les déictiques et l'ellipse.

Nous sommes d'accord pour définir la vicariance par la fonction de remplacement qu'elle assume. C'est cette fonction de remplacement qui va nous permettre de distinguer d'emblée la vicariance, les déictiques et l'anaphore d'une part de l'ellipse d'autre part.

H. Bonnard définit l'ellipse comme "la suppression d'un mot entier ou de plusieurs mots" (1981: 35). A cela il faut ajouter que les mots supprimés sont restituables pour comprendre la phrase et que la pensée les restitue nécessairement (nous disons bien la pensée et non le discours: en effet certaines ellipses peuvent ne pas être nécessairement restituées de manière linguistique, comme le note Onana Atouba, 2006: 1425). Or, on voit que l'ellipse fonctionne en réalité non pas comme un remplacement comme c'est le cas avec le verbe faire ou l'anaphore, mais comme un ajout, un complément: c'est le cas avec le mot oui que $\mathrm{H}$. Bonnard définit comme vicariant. Lorsque l'on a la question: Est-ce que tu vas venir?, suivie de la réponse: Oui, il faut en réalité ajouter à cet adverbe: je vais venir, qui est implicite. On voit que l'on n'est pas du tout dans le même cas qu'avec la vicariance, où le mot fajre permet de remplacer un autre verbe mais ne fonctionne pas comme un ajout. C'est donc comme une ellipse d'une proposition sous-jacente, positive ou négative, que fonctionnent les mots oui et non, et l'ellipse est en fait une condensation de proposition que compense un ajout par la pensée. Rien de tel avec la vicariance. Dans: “Vous êtes gentil de m'aimer, mais vous le seriez aussi de me tutoyer, comme vous l'aviez promis et comme tu avais commencé de le faire" (Marcel Proust, Du côté de Guermantes, d'après Frantext), le verbe faire remplace un autre verbe et 
son complément, me tutoyer, et ne demande aucun ajout par la pensée, si ce n'est celui qui consiste à récupérer l'antécédent, contrairement à l'ellipse. Nous ne pouvons donc pas d'emblée admettre le mot oui ou le mot non dans la classe des mots vicariants.

Mais le plus important va être pour nous de délimiter la place de la vicariance par rapport d'une part à l'anaphore, et d'autre part aux déictiques.

L'anaphore est généralement définie comme la reprise d'un segment d'une phrase ou d'un texte, qui constitue son antécédent. C'est là une définition sur laquelle semblent être d'accord la majorité des auteurs. Ainsi lit-on dans la Grammaire méthodique du français que "(I)'anaphore se définit traditionnellement comme toute reprise d'un élément antérieur dans un texte", et que "(p)lus précisément, une expression est anaphorique si son interprétation référentielle dépend nécessairement d'une autre expression qui figure dans le texte" (Riegel et al., 2009: 1029). De même, María Dolores Vivero García note que "(I)a anáfora, como se sabe, remite a un segmento textual anterior, estableciendo relaciones de identidad semántica entre unidades textuales discontinuas" ("l'anaphore, comme on le sait, renvoie à un segment textuel antérieur, en établissant des relations d'identité sémantique entre des unités textuelles discontinues') (1997: 533). Selon le Grand Dictionnaire Larousse encyclopédique Linguistique et sciences du langage (2007 : 36), "en grammaire, l'anaphore est un processus syntaxique consistant à reprendre par un segment, un pronom en particulier, un autre segment du discours, un syntagme nominal antérieur, par exemple. Ainsi, il y a anaphore par en dans la phrase: Des vacances, j'en ai vraiment besoin. Il y a anaphore par tous dans femmes, enfants, vieillards, tous étaient venus. Le segment représenté est dit antécédent".

Dans le Nouveau dictionnaire encyclopédique des sciences du langage de Jean-Marie Schaeffer et d'Oswald Ducrot, l'anaphore est dé- finie de la manière suivante (1995: 457):

Un segment de discours est dit anaphorique lorsqu'il fait allusion à un autre segment, bien déterminé, du même discours, sans lequel on ne saurait lui donner une interprétation (même simplement littérale). En reprenant un terme de Tesnière, nous appellerons source sémantique le segment auquel renvoie l'anaphorique (on parle aussi d'interprétant, ou souvent d'antécédent, car il précède généralement l'anaphorique; étymologiquement d'ailleurs, l'anaphore, c'est ce qui reporte en arrière, mais le mot anaphore est pris dans cet article avec un sens plus général, qui comprend, comme cas particulier, la cataphore, c'est-à-dire l'allusion à un segment textuel ultérieur, comme dans l'exemple (1) ci-dessous). L'anaphorique et sa source peuvent appartenir soit au même énoncé, soit à deux énoncés successifs. Ainsi l'anaphore joue un double rôle elle intervient dans la combinatoire sémantique interne à la phrase, mais elle engage aussi la phrase dans les relations transphrastiques constituant le texte. Dans les exemples qui suivent l'anaphorique est en italiques, et sa source en majuscules:

(1) S'il vient, PIERRE sera content.

(2) J'AI RENCONTRE DES AMIS, ces amis (ils, qui) m'ont parlé de toi.

(3) Jean M'A DIT QU'IL FERAIT BEAU. Jacques aussi.

(4) Jean connaît ma MAISON, mais pas la tienne.

(5) JEAN DETESTE PAUL, et inversement (l'inverse).

(6) JEAN, PAUL ET JACQUES sont venus. Tous étaient contents (Aucun n'était content).

(7) J'AI APPELE UN TAXI, mais ce taxi était occupé.

(8) TA VOITURE est belle, mais les sièges sont durs.

On voit là que divers types d'anaphores sont évoquées, aussi bien des anaphores classiques comme en (2) que des anaphores résomptives comme en (3), que nous considérerions pour notre part comme des ellipses, ou des anaphores associatives comme en (8). Adriana Da Silva, dans le passage qui suit, nous redonne une définition traditionnelle de l'anaphore et nous permet de voir en quoi consiste précisément un autre type d'anaphore, l'anaphore conceptuelle, laquelle 
récupère son antécédent d'une manière particulière (Da Silva, 2008 : 266): "Inicialmente, a anáfora foi considerada uma espécie de substituta de um item lexical antecedente que se retomava no texto, mas há casos, como o das anáforas conceituais, nos quais as anáforas não reativam referentes previamente apresentados no texto" ('Au début, l'anaphore a été considérée comme une espèce de substitut d'une unité lexicale antécédente qui était reprise dans le texte, mais il y a des cas, comme celui des anaphores conceptuelles, dans lesquels les anaphores ne réactivent pas des référents précédemment présentés dans le texte'). Et l'auteur écrit un peu plus Ioin (2008: 267):

Como já afirmamos, a anáfora é um fenômeno lingüístico que permite ao leitor estabelecer uma relação semântica entre itens lexicais de um texto. Dessa forma, ela o ajuda a compreender as relações entre as partes que formam o texto como um todo significativo, possibilitando-Ihe construir a coêrencia textual. Exemplo de retomada:

(1) O jornalista acompanhou os times ao estádio. Eles fizeram uma partida importante.

Em (1), por exemplo, a anáfora Eles retoma o antecedente os times e concorda com ele em pessoa e número. Em outras palavras, essa anáfora está se referindo explicitamente ao SN antecedente, de forma que podemos concluir que os times fizeram uma partida. Em (1), a anáfora e o antecedente são correferenciais. Trata-se, portanto, de uma anáfora pronominal (AP). Já em (2) há um exemplo classico de AC.

(2) O jornalista acompanhou o time ao estádio. Eles fizeram uma partida importante.

Em (2) não podemos dizer que a anáfora Eles retoma diretamente o SN o time, pois não há concordancia de número entre a anáfora e o seu antecedente. Nesse caso, a anáfora remete ao antecedente o time, não se referindo a ele propriamente. Tanto é que não podemos substituir Eles por o time na seqüência textual. Na verdade, eles se refere aos sujeitos, os seres [+ HUMANOS]que compõem o time, ou seja, os jogadores.
('Comme nous l'avons déjà affirmé, l'anaphore est un phénomène linguistique qui permet au lecteur d'établir une relation sémantique entre les unités lexicales d'un texte. De cette façon, elle l'aide à comprendre les relations entre les parties qui forment le texte, et lui donne la possibilité de construire la cohérence textuelle. Exemple de reprise:

(1) Le journaliste a accompagné les équipes au stade. Elles ont fait un match important.

En (1), par exemple, l'anaphore Elles reprend l'antécédent les équipes et s'accorde avec ce dernier en genre et en nombre. En d'autres termes, cette anaphore se réfère explicitement au SN antécédent, de sorte que l'on peut conclure que les équipes ont fait un match. En (1), l'anaphore et l'antécédent sont co-référentiels. II s'agit, par conséquent, d'une anaphore pronominale (AP). On retrouve en (2) un exemple classique d'anaphore conceptuelle.

(2) Le journaliste a accompagné l'équipe au stade. Ils ont fait un match important.

On ne peut pas dire qu'en (2) l'anaphore I/s reprenne directement le SN l'équipe, puisqu'il n'y a pas d'accord en nombre entre l'anaphore et son antécédent. Dans ce cas, l'anaphore renvoie à l'antécédent l'équipe, en ne se référant pas proprement à lui. De telle sorte que l'on ne peut pas remplacer I/s par l'équipe dans la séquence textuelle. En fait, ils se réfère aux sujets, aux êtres [+ HUMAINS] qui composent l'équipe, c'est-à-dire aux joueurs.')

Nous considérerons également la définition de L. Tesnière, dans la mesure où ce dernier fait rentrer dans la classe des mots anaphoriques le verbe faire. Voyons comment l'auteur des Eléments de syntaxe structurale définit les mots anaphoriques (1969: 90-91):

Le phénomène d'anaphore ne se produisant que dans la phrase organisée, les anaphoriques n'ont par eux-mêmes aucun sens tant qu'ils ne sont pas inclus dans une phrase.

En d'autres termes, les anaphoriques sont des mots vides sur le plan statique. Mais ils deviennent automatiquement pleins sur le plan dyna- 


\section{mique.}

Ainsi l'anaphorique son ne désigne par lui-même personne, et ce n'est que dans une phrase comme par exemple Alfred aime son père qu'il prend le sens d'Alfred.

Les anaphoriques sont donc des mots vides qui ne se réalisent dans la phrase que comme mots pleins. Ce sont des mots pleins virtuels.

[...] Les anaphoriques se classent tout naturellement comme les mots pleins avec lesquels ils s'identifient sémantiquement.

Nous distinguerons donc, parmi les anaphoriques, des substantifs, des adjectifs, des verbes et des adverbes.

Et L. Tesnière donne comme exemples de substantifs anaphoriques le pronom possessif (On voit les maux d'autrui d'un autre œil que les siens (c'est-à-dire ceux de on); Corneille), ou le pronom relatif, comme exemple d'adverbe anaphorique l'adverbe ainsi, et, plus important pour nous, il écrit que "(I)e principal verbe anaphorique est le verbe faire [...]: On regarde une femme savante comme on fait (=comme on regarde) une belle arme. (La Bruyère, Caractères, III)".

Les définitions de l'anaphore que nous avons données insistent toutes à la fois sur la nécessité de reprise d'un segment textuel précédemment mentionné ou à venir pour définir l'anaphore et la cataphore (qu'il sera plus pratique pour nous de considérer comme similaires ici), et sur la diversité des procédés anaphoriques. En somme une seule condition semble nécessaire: qu'il y ait reprise d'un élément textuellement mentionné. Encore cette reprise peut-elle n'être pas nécessairement une reprise identique, comme c'est le cas dans l'anaphore conceptuelle, où l'on peut observer des variations en nombre dans la reprise, ou dans l'anaphore qualifiée d'infidèle par la grammaire traditionnelle.

Quelle est, dès lors, la différence entre anaphore, déictique et vicariance, ou en quoi la vicariance constitue-t-elle un type particulier de déictique ou d'anaphore? En ce qui concerne la première distinction, on s'en tiendra à des considérations générales, par exemple celles formulées par André Martinet dans sa Syntaxe générale (1985: 127):

On connaît la distinction établie traditionnellement, pour certains pronoms, entre leurs emplois anaphoriques et leurs emplois déictiques un démonstratif s'emploie de façon anaphorique s'il renvoie à une entité mentionnée dans ce qui précède dans le discours; son emploi est déictique s'il fait référence à un élément de la situation. La distinction est nette dans les emplois de deux démonstratifs qui s'opposent pour marquer, l'un la proximité, l'autre la distance : en référence au contexte précédent, celui-ci désignera l'élément d'une coordination qui est le plus rapproché dans le discours, celui-là, celui qui est le plus éloigné. En référence à la situation, celuici désignera l'objet le plus proche dans l'espace, celui-là celui qui est le plus loin. Dans l'emploi qui est fait de ceci pour présenter ce qui va suivre dans le discours, on perçoit ce qui relie les emplois anaphoriques et les emplois déictiques.

En fait, la distinction entre la référence au contexte et la référence à la situation est souvent utile pour comprendre la structuration des systèmes pronominaux. La référence à la situation est claire dans le cas des pronoms dits "personnels": c'est la situation qui indique qui est je, celui qui parle, qui est tu, celui à qui l'on s'adresse; dans les systèmes qui présentent plus d'une "troisième personne", on trouvera la distinction entre ce qui est présent et visible et ce qui ne l'est pas, donc de nouveau référence à la situation. Il en va de même pour les pronoms possessifs dont on attend qu'ils offrent un système calqué sur celui des personnels. A l'opposé, les pronoms relatifs sont sous la dépendance stricte et étroite du contexte, le relatif renvoyant à un antécédent. Se réfèrent à un élément du contexte ceux que nous avons désignés comme les quantitatifs; plusieurs, par exemple, dans j'en ai lu plusieurs en référence aux livres prémentionnés d'un auteur. Mais on dira très bien, en situation, quand on désigne des pommes dans un étalage, J'en veux plusieurs. Les interrogatifs réclament un référent qui va être fourni, en réponse, soit dans 
un contexte: Qui...?, Un homme qui fera l'affaire, soit dans la situation : Oú... ? Ici ! Echappent au cadre de cette opposition, les pronoms que nous désignons comme indépendants : quelqu’un, par exemple, ne renvoie à personne qui ait été présenté ou désigné antérieurement.

Alors que les déictiques assument donc une fonction de substitution qui est compréhensible à partir du seul contexte d'énonciation, les anaphores, au contraire, font nécessairement appel à un élément précédemment mentionné dans la chaîne parlée. Autrement dit, le référent des déictiques est lié à l'ici/maintenant et relève de la catégorie du discours, pour reprendre le paradigme de Benveniste, alors que l'anaphore relèverait du récit, de la troisième personne. On notera également que le référent des déictiques est implicite et donné par le contexte, donc non clairement formulé, alors qu'au contraire le référent de l'anaphore est donné par les informations de la phrase ou du texte et doit être mentionné. Des paramètres d'ordre pragmatique, comme ceux évoqués par André Martinet, interviennent également selon chaque situation d'énonciation.

Quelle est la situation de la vicariance par rapport aux déictiques et à l'anaphore?

\section{Conditions de la vicariance}

On pourrait dans un premier temps se demander si les anaphores et les déictiques d'une part, et la vicariance d'autre part, fonctionnent sur un même mode de récupération du référent. Il apparaît dès lors que la vicariance semble être sur ce point identique à l'anaphore et à la cataphore, puisqu'elle peut aussi bien annoncer son référent que le suivre, comme dans: "Vous êtes gentil de m'aimer, mais vous le seriez aussi de me tutoyer, comme vous l'aviez promis et comme tu avais commencé de le faire" (Marcel Proust, Du côté de Guermantes, op. cit), ou dans la phrase qui suit, où le verbe faire annonce au contraire ce qu'il va remplacer, boire un café maintenant: ce que je te propose de faire, c'est de boire un café maintenant.
Le mode de récupération du référent nous sert donc à différencier l'anaphore et la vicariance des déictiques: dans le premier cas la référence est clairement mentionnée dans l'entourage textuel, dans le second elle est récupérable à partir de la seule situation d'énonciation. La vicariance est donc un type d'anaphore, puisqu'elle a un antécédent. II reste à voir comment la caractériser.

Considérons l'aspect sémantique de la vicariance. Si l'on observe les mots vicariants prototypiques que sont faire et que, on constate tout d'abord qu'il s'agit de mots qui ont un sémantisme très vague et très abstrait qui leur permet de reprendre un grand nombre d'éléments au niveau du discours. Ces mots sont bien des mots "super-inclusifs", pour citer Bernard Pottier (op. cit.): faire peut récupérer tous les noms d'activité lorsqu'il est vicariant, et que vicariant peut quant à lui répéter n'importe quel rapport circonstanciel, par exemple un rapport de cause dans la phrase qui suit: "Comme les langues germaniques actuellement parlées se développent indépendamment les unes des autres depuis de longs siècles et que leur évolution n'a presque jamais cessé d'être rapide, que même à certains moments elle s'est précipitée, ces langues sont maintenant très différentes les unes des autres" (Meillet, 1937 : 16-17).

Toutes les anaphores ont-elles une virtualité sémantique aussi importante? On voit que non si l'on observe un grand nombre d'anaphores infidèles traditionnelles, d'anaphores associatives ou d'anaphores conceptuelles. Dans une anaphore comme: J'ai vu Max. Ce garçon est vraiment gentil, on voit par exemple que le mot anaphorique ce a besoin d'un déterminé qui a un sémantisme précis, lequel ne pourrait pas être rapproché du sémantisme vague d'un mot comme faire ou d'une conjonction comme que.

On relève ensuite que lorsque l'on reprend un verbe avec faire ou une conjonction avec que, le contenu notionnel du verbe ou de la conjonc- 
tion repris reste le même dans la reprise. C'est ce qui nous permet de voir que la vicariance garde une identité sémantique parfaite avec son antécédent et ne lui ajoute aucune information nouvelle (Jacquelin Pinchon (1972) parle d' "anaphore convergente” pour désigner le même phénomène à propos des pronoms). En effet, que ce soit avec le verbe faire ou avec la conjonction que, il n'y a ni recatégorisation ni redéfinition de l'antécédent, dont le contenu notionnel ne varie pas. C'est ce qui nous permet donc de ne pas considérer comme vicariantes aussi bien les anaphores conceptuelles, qui changent une caractéristique du nombre de l'antécédent, que les anaphores associatives, qui introduisent une anaphore qui ne correspond qu'à une partie de leur antécédent, ou que les anaphores introduites par un déterminant démonstratif ou un article défini. Ces deux derniers types d'anaphore, en effet, ne reprennent pas le contenu sémantique de leur antécédent de façon neutre. C'est ce que l'on voit dans des cas comme: Le suédois est parlé en Suède et en Finlande, mais cette belle langue est malheureusement peu connue en dehors de ces pays, ou: Le suédois a emprunté de nombreux mots au français. En effet, la langue de Molière était et continue à être très appréciée en Suède. Dans la première phrase, l'anaphore cette belle langue ajoute des sèmes à son antécédent, le suédois, et dans le deuxième cas, l'anaphore la langue de Molière redéfinit le français et adopte un point de vue particulier sur l'antécédent, considéré ici à travers une expression stéréotypique qui ne l'envisage plus seulement comme le français mais comme la langue qu'a, entre autres, utilisée un auteur représentatif de la littérature française.

On voit donc que les mots vicariants se définissent essentiellement sémantiquement, à la fois par leur super-inclusivité et par leur identité sémantique avec leur antécédent. II convient à présent de déterminer quels sont les mots que l'on peut considérer comme vicariants.

Il apparaît tout d'abord que les intuitions de
Gérard Moignet et de Bernard Pottier ainsi que l'affirmation de Georges Kleiber au sujet du mot chose sont parfaitement fondées. Voyons en quoi les mots chose, truc, bidule et machin sont vicariants. Ces mots ont un sémantisme vague qui leur permet de remplacer un très grand nombre d'éléments. En effet, une chose, ou quelque chose, peut renvoyer aussi bien à des objets concrets (Une bouilloire, c'est quelque chose de pratique) qu'à des entités abstraites (Le suédois est une chose que je rêve d'apprendre), et s'il convient de voir la spécificité de bidule ou machin, on peut noter que ces mots peuvent remplacer n’importe quel nom d'objet matériel.

Bernard Pottier va plus loin et rapproche faire du pronom interrogatif qui ou quoi. Effectivement, qui et quoi, comme tous les pronoms interrogatifs et tous les pronoms relatifs, fonctionnent bien comme des substituts qui ont un sémantisme vague et qui sont sémantiquement identiques au mot qu'ils annoncent ou qu'ils reprennent.

On retrouve également, parmi les mots vicariants les plus importants en français, les pronoms personnels de troisième personne, le pronom $y$, les pronoms ceci/cela et leur paradigme, et les pronoms tous et tout, qui tous se caractérisent par leur super-inclusivité et leur neutralité sémantique.

Certains mots, enfin, sont occasionnellement vicariants. Tel est le cas de en, qui peut ne pas reprendre parfaitement son antécédent ou qui peut au contraire être sémantiquement identique à ce dernier. Si l'on observe tout d'abord une phrase comme: /l y a du café. Est-ce que tu en veux?, le pronom en paraît garder le sémantisme complet de café et être identique à ce dernier. Mais cette impression provient du fait que l'antécédent est un nom massif. Si l'on observe en effet la phrase: J'ai beaucoup de livres de cet auteur. Est-ce que tu veux que je t'en prête?, on voit que en ne reprend qu'une partie de l'antécédent et qu'il ne prélève qu'un certain nombre de livres. Ce pronom n'est donc pas sé- 
mantiquement identique à l'antécédent qui est le sien dans ce cas. Au contraire, en est vicariant et reprend de façon complète son antécédent dans: Ai-je jamais connu une aussi longue suite ininterrompue de beaux jours? Certains matins sont si glorieusement purs que l'on ne sait qu'en faire (Gide, Journal, cité par Pinchon, 1972 : 44).

\section{Conclusion}

De deux mots vicariants au départ, nous en arrivons à un ensemble de mots vicariants assez hétérogène, puisque nous avons aussi bien des verbes que des pronoms vicariants. Mais la catégorie de départ était elle-même hétérogène, étant donné que l'on rencontrait aussi bien dans les mots vicariants prototypiques un verbe (faire) qu'une conjonction (que) et, éventuellement, un nom (chose). II n'était donc pas possible de trouver une définition syntaxique de la vicariance, et seule une définition sémantique pouvait être envisagée. Dans cette perspective, les mots vicariants peuvent être définis comme des mots au sémantisme abstrait et sémantiquement identiques avec leur antécédent.

\section{Bibliographie citée}

Battaglia, Salvatore, 1964: Grande dizionario della lingua italiana, Torino : Editrice Torinese.

Bonnard, Henri, 1981 : Code du français courant, Paris : Magnard.

Brunot, Ferdinand, 1936 : La pensée et la langue, Paris: Masson et Cie Editeurs.

DA Silva, Adriana, 2008: "A leitura e o processamento da anáfora conceitual", Linguagem em (Dis)curso 8, 265-287.

Dubols, Jean et al., 2007 : Grand Dictionnaire Larousse encyclopédique Linguistique et sciences du langage, Paris : Larousse.

ERIKSSON, Olof, 1985: La suppléance verbale en français moderne, Göteborg : Acta universitatis Gothoburgensis.
Grevisse, Maurice, 1970 : Problèmes de langage, tome 5, Gembloux: Editions J. Duculot.

GuIlbert, Louis, René Lagane et Georges Niobey, 1978: Grand Larousse de la langue française, Paris: Librairie Larousse.

HJelmslev, Louis, 1971: Essais linguistiques, Paris: Editions de Minuit.

Instituto Antônio houalss De Lexicografola Portugal, 2003 : Dicionário Houaiss da Língua Portuguesa, Lisboa: Círculo Leitores.

KLEIBER, Georges, 1987 : "Mais à quoi sert donc le mot chose?", Langue française 73, 109-128.

LARDON, Sabine et Marie-Claire THOMINe, 2009 Grammaire du français de la Renaissance. Etude morphosyntaxique, Paris: Editions classiques Garnier.

Le BIDoIs, Georges et Robert Le BIDoIs, 1968 : Syntaxe du français moderne, tome 2, Paris : Editions A. et J. Picard et Cie.

Martinet, André, 1985 : Syntaxe générale, Paris : Armand Colin.

Meyer-Lübke, Wilhelm, 1900 : Grammaire des langues romanes, tome 3, Paris: H. Welter.

Moıgnet, Gérard, 1974 : Etudes de psycho-systématique française, Paris : Klincksieck.

NøJGAARD, Morten, 1970 : "Notes sur que reprenant si", Revue romane 5, 120-129.

Onana Atouba, Pierre Paulin, 2006 : "Sobre la elispsis pragmática", dans Actas del 35 Simposio Internacional de Lingüística Española.

Pinchon, Jacqueline, 1972 : Les pronoms adverbiaux EN et $Y$ : problèmes généraux de la représentation pronominale, Genève : Droz.

Ponchon, Thierry, 1994 : Sémantique lexicale et 
sémantique grammaticale : le verbe faire en français médiéval, Genève: Droz.

Pottier, Bernard, 1967 : "Présentation de la linguistique. Fondements d'une théorie", Travaux de linguistique et de littérature 5, 7-60.

Riegel, Martin, Jean-Christophe Pellat et René Rıoul, 2009: Grammaire méthodique du français, Paris: PUF.

Robert, Paul, Josette Rey-Debove et Alain Rey, 2008 : Nouveau Petit Robert, Paris : Le Robert.

Schaeffer, Jean-Marie et Oswald Ducrot, 1995 : Nouveau dictionnaire encyclopédique des sciences du langage, Paris : Le Seuil.

Soutet, Olivier, 1992 : Etudes d'ancien et de moyen français, Paris : PUF.

Tesniere, Lucien, 1969 : Eléments de syntaxe structurale, Paris : Klincksieck.

VerJans, Thomas, 2007 : "Sur un emploi de que : le que " vicariant »", Cahiers de linguistique analogique 4 , 133-155.

Vivero García, María Dolores, 1997 : "La anáfora desde una perspectiva textual", Thélème. Revista complutense de estudios franceses 12, 533-544.

\subsection{Oeuvres citées}

Melllet, Antoine, 1937: Caractères généraux des langues germaniques, Paris: Librairie Hachette.

\subsection{Corpus informatiques utilisés}

Laboratoire d'Analyse et dE Traitement Informatique de la Langue Française: Frantext, CNRS/Université de Loraine [www.frantext.fr, date de consultation: 28/09/2013]. 